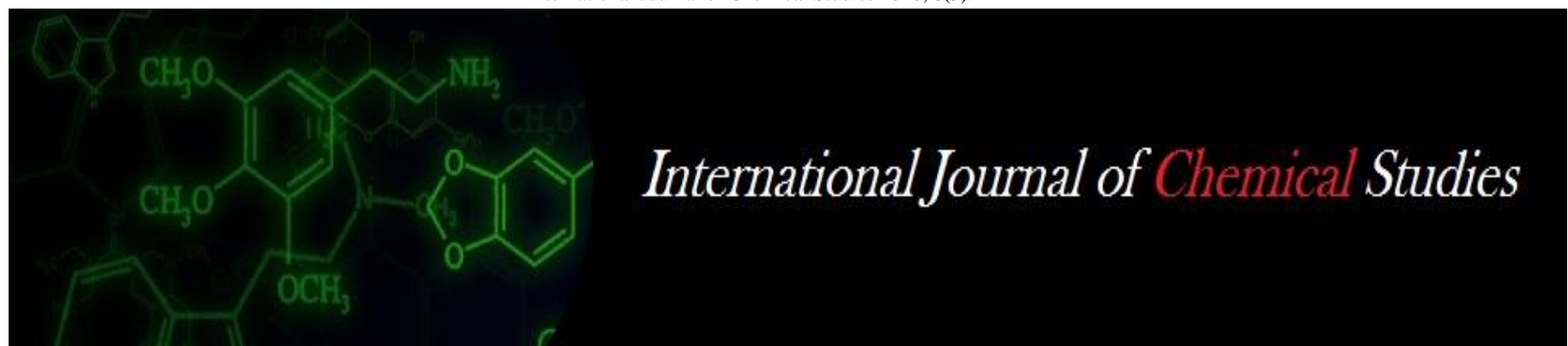

P-ISSN: 2349-8528

E-ISSN: 2321-4902

www.chemijournal.com

IJCS 2020; 8(5): 211-214

(C) 2020 IJCS

Received: 12-06-2020

Accepted: 03-08-2020

\section{Rashid Ashraf}

Department of Agronomy, Bihar Agricultural University Sabour,

Bhagalpur, Bihar, India

\section{MK Singh}

Department of Agronomy, Bihar Agricultural University Sabour,

Bhagalpur, Bihar, India

S Tyagi

Department of Agronomy, Bihar Agricultural University Sabour,

Bhagalpur, Bihar, India

\section{Arun Kumar}

Department of Agronomy, Bihar Agricultural University Sabour, Bhagalpur, Bihar, India

Shweta Shambhavi

Department of Soil Science and Agricultural Chemistry, Bihar Agricultural University Sabour, Bhagalpur, Bihar, India

Corresponding Author:

\section{MK Singh}

Department of Agronomy, Bihar Agricultural University Sabour,

Bhagalpur, Bihar, India

\section{Effect of weed management practices on transplanted finger millet (Eleusine coracana $\mathrm{L}$.) in Bihar}

\author{
MD Rashid Ashrafi, MK Singh, S Tyagi, Arun Kumar and Shweta \\ Shambhavi
}

DOI: https://doi.org/10.22271/chemi.2020.v8.i5c.10302

\begin{abstract}
A field experiment was conducted at Research Farmland, Bihar Agricultural University, Sabour, Bhagalpur, Bihar during kharif season of 2019 to evaluate weed management practices in transplanted finger millet. The experiment was laid out in randomized block design with three replication and consisting eleven treatment viz., $\mathrm{T}_{1}$ (Weedy check), $\mathrm{T}_{2}$ (Hoeing at 20 and $40 \mathrm{DAT}$ ), $\mathrm{T}_{3}$ (Pendimethalin @ $750 \mathrm{~g}$ ai ha $^{-1}$ as pre-emergence), $\mathrm{T}_{4}\left(2,4-\mathrm{D} @ 750 \mathrm{~g}\right.$ ai ha $^{-1}$ as post-emergence), $\mathrm{T}_{5}$ (Bispyribac sodium @ $20 \mathrm{~g}_{\text {ai ha }}{ }^{-1}$ as post-emergence), T6 (Pendimethalin @ $750 \mathrm{~g}^{2} \mathrm{ha}^{-1}$ as pre-emergence $f b$ 2, 4-D @ 750 $\mathrm{g}$ ai ha $\mathrm{h}^{-1}$ as post-emergence), $\mathrm{T}_{7}$ (Pendimethalin @ $750 \mathrm{~g}_{\text {ai ha }}{ }^{-1}$ as pre-emergence $f b$ Bispyribac sodium @ $20 \mathrm{~g}_{\text {ai ha }}{ }^{-1}$ as post-emergence), T8 (Pretilachlor @1000 $\mathrm{g}^{-1}$ ha $^{-1}$ as pre-emergence), $\mathrm{T}_{9}$ (Pretilachlor @ $1000 \mathrm{~g}_{\text {ai ha }}{ }^{-1}$ as pre-emergence $f b 2$ 2, 4- D @ $750 \mathrm{~g}$ ai ha- ${ }^{-1}$ as post- emergence), $\mathrm{T}_{10}$ (Pretilachlor @ 1000 $\mathrm{g}$ ai ha ${ }^{-1}$ as pre- emergence $f b$ Bispyribac sodium @ $20 \mathrm{~g}$ ai ha ${ }^{-1}$ as post- emergence) and $\mathrm{T}_{11}$ (weed free). The results revealed that sequential application of Pretilachlor @1000 g / Pendimethalin@750 g ai ha-1 as pre-em $f b$ Bispyribac sodium @ $20 \mathrm{~g}$ ai ha ${ }^{-1}$ as post-emergence for higher yield, net return and B:C ratio of transplanted finger millet in Bihar.
\end{abstract}

Keywords: Weed management, finger millet, growth, yield, economics

\section{Introduction}

Selective utilization of crops and varieties in recent times have threatened agrobiodiversity leading to rapid erosion of natural resources and consequently affecting nutritional security. One of the possible pathways for conservation of such neglected agrobiodiversity resources is to bring them into use thereby making them viable crops within the contemporary social and economic context. Climate change portends less and erratic rain, more heat, reduced water availability and increased malnutrition. Under such situation finger millet crop can withstand these challenges and produce multiple securities (food, fodder, health, nutrition, livelihood and ecological). All these qualities of millet farming system make them climate change compliant crops and helping in mitigation of climate change. Basically finger millet (Eleusine coracana) is extreme drought tolerant tropical crop mostly suitable for dry regions. Finger millet (Eleusine coracana L.) is an important rainfed crop grown in India. It is commonly known as Ragi or Madua. In India, it is cultivated in an area of 1.02 million ha with a production of 1.39 million tonne. (Anonymous, 2017) ${ }^{[1]}$. Weed infestation is one of the serious constraints in ragi production due to slower initial growth. This situation causes higher competition and may result in drastic reduction in grain yield. Early period up to four weeks after transplanting, yield is reduced drastically. Delayed weeding will be less effective because sufficient damage would have occurred at the critical period. In present days, apart from expensive labour charges, timely availability of labour is a limitation for undertaking cultural operations like hand weeding. In such situations, suitable technology with less labour requirement will be most helpful to farmers for controlling weeds effectively and chemical weed control is one of such measures. Hence, present study was conducted to know the effect of weed management practices on growth and yield in finger millet.

\section{Material and Methods}

A experiment was carried out at Research farm, Bihar Agricultural University, Sabour during kharif season of 2019 under rainfed condition. 
The farm is situated at $25^{\circ} 50^{\prime} \mathrm{N}$ latitude, $87^{\circ} 19^{\prime} \mathrm{E}$ longitude and at an altitude of $52.73 \mathrm{~m}$ above mean sea level. The sandy-loam soil of the experimental field was low in organic carbon $(0.48 \%)$ and available $\mathrm{N}\left(215 \mathrm{~kg} \mathrm{ha}^{-1}\right)$, medium in available $\mathrm{P}\left(23.5 \mathrm{~kg} \mathrm{ha}^{-1}\right)$ and $\mathrm{K}\left(183.6 \mathrm{~kg} \mathrm{ha}^{-1}\right)$ with $\mathrm{pH} 7.35$. The experiment was laid out in randomized block design with three replication and consisting eleven treatment viz., $\mathrm{T}_{1}$ (Weedy check), $\mathrm{T}_{2}$ (Hoeing at 20 and 40 DAT), $\mathrm{T}_{3}$ (Pendimethalin @ $750 \mathrm{~g} \mathrm{ai} \mathrm{ha}^{-1}$ as pre-emergence), $\mathrm{T}_{4}$ (2, 4- D @ $750 \mathrm{~g}_{\text {ai ha }}{ }^{-1}$ as post-emergence), $\mathrm{T}_{5}$ (Bispyribac sodium @ $20 \mathrm{~g}_{\text {ai ha }}{ }^{-1}$ as post-emergence), $\mathrm{T}_{6}$ (Pendimethalin @750 g ai $\mathrm{ha}^{-1}$ as pre-emergence $f b$ 2, 4-D @ $750 \mathrm{~g}$ ai ha $\mathrm{ha}^{-1}$ as postemergence), $\mathrm{T}_{7}$ (Pendimethalin @750 $\mathrm{g}$ ai ha- $\mathrm{ha}^{-1}$ as preemergence $f b$ Bispyribac sodium @ $20 \mathrm{~g}^{\text {ai ha }} \mathrm{ha}^{-1}$ as postemergence), $\mathrm{T}_{8}$ (Pretilachlor @1000 g ai ha $\mathrm{ga}^{-1}$ as preemergence), $\mathrm{T}_{9}$ (Pretilachlor @1000 $\mathrm{g}$ ai ha ${ }^{-1}$ as preemergence $f b$ 2, 4- D @ $750 \mathrm{~g}$ ai ha ${ }^{-1}$ as post- emergence), $\mathrm{T}_{10}$ (Pretilachlor @ $1000 \mathrm{~g}$ ai ha ${ }^{-1}$ as pre- emergence $f b$ Bispyribac sodium@20 g ai ha ${ }^{-1}$ as post- emergence) and $\mathrm{T}_{11}$ (weed free). The variety used for the experiment was GPU-67 with a spacing of $20 \times 20 \mathrm{~cm}$. A recommended dose of fertilizers (50:40:25 N: $\mathrm{P}_{2} \mathrm{O}_{5}: \mathrm{K}_{2} \mathrm{O} \mathrm{kg} / \mathrm{ha}$ ) was applied equally to each plot. Nitrogen was applied in two splits. Full amount of phosphate and potassic fertilizer and half amount of nitrogenous fertilizer were applied as uniformly as possible before transplanting. The rest half of the nitrogenous fertilizer was applied as top dressing during the time of tillering and finger initiation stage of crop. The source for nitrogen, phosphorous and potassium were urea, SSP and MOP, respectively. The experimental data recorded for growth, yield attributes and yield were statistically analysed statistically analyzed by the procedure of analysis of variance for randomized block design (RBD) given by Panse and Sukhatma (1985). For significant ' $F$ ' test, critical difference (CD) was reported at 5 per cent probability level.

\section{Results and Discussion}

\subsection{Effects weed management practices on growth attributes}

Weed management practices significantly enhanced the growth attributes of finger millet over weedy check (Table 1). The tallest plant $(109.11 \mathrm{~cm})$ was recorded with weed free treatment which remain at par with hoeing at 20 and 40 DAT. Highest leaf area index (3.61) was recorded with weed free treatment while it was on par with hoeing at 20 and 40 DAT, 5 Bispyribac sodium@20 g ai ha ${ }^{-1}$, Pendimethalin@750 g ai ha $^{-1} f b$ Bispyribac sodium @ $20 \mathrm{~g} \mathrm{ai} \mathrm{ha}^{-1}$ as well as Pretilachlor@1000 g ai ha ${ }^{-1}$ fb Bispyribac sodium @ $20 \mathrm{~g}$ ai $\mathrm{ha}^{-1}$ applied plots. Similar results were reported by Kumar et al., (2015) ${ }^{[10]}$ and Prithvi et al., (2015) ${ }^{[2]}$. More number of tillers per hill $\left(6.44\right.$ hill $^{-1}$ at harvest) were recorded with application of Pretilachlor @1000 g ai ha ${ }^{-1} \mathrm{fb}$ Bispyribac sodium @20 g ai ha ${ }^{-1}$ which at par with Bispyribac sodium @ $20 \mathrm{~g}$ ai ha ${ }^{-1}$ and Pendimethalin@750 g ai ha ${ }^{-1} \mathrm{fb}$ Bispyribac sodium @20 g ai ha ${ }^{-1}$. Maximum dry matter accumulation (32.50 $\mathrm{g} \mathrm{hill}^{-1}$ ) was recorded with weed free plots which was on par with Hoeing at 20 and 40 DAT, Pendimethalin @ 750 $\mathrm{g}$ ai ha ${ }^{-1} f b$ 2, 4-D@750 g ai ha ${ }^{-1}$, Pendimethalin@750 g ai $\mathrm{ha}^{-1}$ as $f b$ Bispyribac sodium @ $20 \mathrm{~g}$ ai ha $\mathrm{g}^{-1}$, Pretilachlor @ $1000 \mathrm{~g}$ ai ha $\mathrm{h}^{-1} \mathrm{fb} 2$ 2, 4- D @ $750 \mathrm{~g}$ ai ha ${ }^{-1}$, and Pretilachlor @1000 g ai ha ${ }^{-1} f b$ Bispyribac sodium @ $20 \mathrm{~g}$ ai ha-1. Similar observation was noticed by Satish et al. (2018) ${ }^{[4]}$ and Kumar et al. (2015) ${ }^{[10]}$. The possible reason of above result might be due to better weed control and congenial environment for maximum utilization of resources like sunlight, nutrients, moisture which helped plant for vigorous growth. Similar trend also reported by Pradhan et al. (2010) ${ }^{[3]}$.

3.2 Effects weed management practices on yield attributes Different weed management practices significantly increased yield attributes of finger millet. Significantly highest number of ear $\mathrm{m}^{-2}$ (158.67) was recorded with application of Pretilachlor@1000 g ai ha ${ }^{-1}$ as pre-em $f b$ Bispyribac sodium @ $20 \mathrm{~g}$ ai $\mathrm{ha}^{-1}$ as post- em which remained at par with Bispyribac sodium @ $20 \mathrm{~g}$ ai ha ${ }^{-1}$ as post-em, and Pendimethalin@750 g ai ha ${ }^{-1}$ as pre-em $f b$ Bispyribac sodium @ $20 \mathrm{~g}$ ai ha ${ }^{-1}$ as post-emergence. Similar result was noticed by Kumar et al. (2015) ${ }^{[10]}$. Highest ear weight (8.52 g) was recorded with Weed free treatment and remained at par with all treatments except Weedy check (Control), Pendimethalin @ $750 \mathrm{~g}$ ai ha ${ }^{-1}$ as pre-em and 2, 4- D @ $750 \mathrm{~g}$ ai ha ${ }^{-1}$ as postemergence. Significantly highest number of finger ear $^{-1}(7.22)$ and test weight $(4.53 \mathrm{~g})$ was recorded with Weed free treatment and remain at par with all treatment except Weedy check (Control). Tuti et al. (2016) ${ }^{[5]}$ observed similar result. Longest finger $(8.90 \mathrm{~cm})$ was recorded with Weed free treatment and remain at par with all treatment except Pendimethalin@750 g ai ha ${ }^{-1}$ as pre-em),2,4-D@750 g ai $\mathrm{ha}^{-1}$ as post-em, and 2, 4- D @ $750 \mathrm{~g}$ ai ha ${ }^{-1}$ as postemergence treatment. Such outcome of different weed management practices on yield attributes may be due to better weed control throughout crop period and efficient nutrient uptake, better utilization of resources and better assimilation and translocation of photosynthates.

\subsection{Effects weed management practices on grain and straw yield}

All most weed control treatments were significantly superior to weedy check in increasing grain yield. Among different weed management practices, weed free plot recorded significantly higher grain and straw yield (23.12 $\mathrm{qha}^{-1}$ and $44.01 \mathrm{qha}^{-1}$, respectively) of transplanted finger millet, which was on par with Hoeing at 20 and 40 DAT, Pendimethalin @ $750 \mathrm{~g}$ ai ha ${ }^{-1}$ as pre-em $f b$ Bispyribac sodium @ $20 \mathrm{~g}$ ai ha ${ }^{-1}$ as post-em, and Pretilachlor @ $1000 \mathrm{~g}$ ai ha ${ }^{-1}$ as pre-em $f b$ Bispyribac sodium @ $20 \mathrm{~g}_{\text {ai ha }} \mathrm{ha}^{-1}$ as post- em. This might be due to effective weed control which results in lower weed population and weed biomass and created favourable condition for crop to produce growth characters and high LAI leads to higher dry matter production and finally higher yield. Satish et al. (2018) ${ }^{[4]}$, Kumar et al. (2017), Prithvi et al. $(2015)^{[2]}$ and Naik et al. (2001) reported similar result.

\subsection{Effects weed management practices on economics}

Significantly higher net return and B: C ratio (Rs 52253 and 1.70 respectively) was found with application of Pretilachlor @ $1000 \mathrm{~g}$ ai ha ${ }^{-1}$ as pre-em $f b$ Bispyribac sodium @ $20 \mathrm{~g}$ ai ha 1 as post-emergence which at par (Rs 52242 and 1.69 respectively) with Pendimethalin @750 g ai ha ${ }^{-1}$ as pre-em $f b$ Bispyribac sodium @ $20 \mathrm{~g}$ ai ha ${ }^{-1}$ as post-emergence application. This might be due the lower weed density and higher yield associated with the respective treatment results higher economics of transplanted finger millet. The similar results were also reported by Kunjur et al. (2018) ${ }^{[8]}$ and Pradhan et al. (2010) ${ }^{[3]}$ in finger millet

\section{Conclusion}

Based on finding of investigation, the results revealed that sequential application of Pretilachlor @1000 g ai ha-1/ Pendimethalin@750 g ai ha- ${ }^{-1}$ as pre-emergence $f b$ Bispyribac sodium @20 g ai ha ${ }^{-1}$ as post-emergence for higher yield, net return and $\mathrm{B}: \mathrm{C}$ ratio of transplanted finger millet in Bihar. 
Table 1: Effect of weed management practices on growth attributes of transplanted finger millet

\begin{tabular}{|c|c|c|c|c|}
\hline \multirow{2}{*}{ Treatments } & \multicolumn{4}{|c|}{ Growth attributes } \\
\hline & Plant height (cm) & LAI & Number of tillers hill-1 & Dry matter accumulation (g hill-1) \\
\hline $\mathrm{T}_{1}$ Weedy check (Control) & 92.51 & 2.62 & 3.77 & 25.70 \\
\hline $\mathrm{T}_{2}$ Hoeing at 20 and 40 DAT & 105.67 & 3.50 & 4.64 & 31.38 \\
\hline $\mathrm{T}_{3}$ Pendimethalin @ $750 \mathrm{~g}$ ai ha ${ }^{-1}$ as pre-em & 101.77 & 3.03 & 4.33 & 28.15 \\
\hline $\mathrm{T}_{4} 2,4-\mathrm{D} @ 750 \mathrm{~g}$ ai ha ${ }^{-1}$ as post-em & 98.17 & 2.94 & 5.56 & 28.22 \\
\hline T5 Bispyribac sodium @ $20 \mathrm{~g}_{\text {ai ha }}{ }^{-1}$ as post-em & 96.67 & 3.25 & 6.00 & 27.81 \\
\hline $\begin{array}{c}\mathrm{T}_{6} \text { Pendimethalin @ } 750 \mathrm{~g} \text { ai ha-1 }^{-1} \text { as pre-em } f b 2, \\
\text { 4-D @ } 750 \mathrm{~g} \mathrm{ai} \mathrm{ha}^{-1} \text { as post-em }\end{array}$ & 98.76 & 2.98 & 5.56 & 29.43 \\
\hline $\begin{array}{l}\mathrm{T}_{7} \text { Pendimethalin @ } 750 \mathrm{~g} \text { ai ha } \mathrm{h}^{-1} \text { as pre-em } f b \\
\text { Bispyribac sodium @ } 20 \mathrm{~g}^{2} \text { ai ha }^{-1} \text { as post-em }\end{array}$ & 94.93 & 3.42 & 6.33 & 29.69 \\
\hline T8 Pretilachlor @ $1000 \mathrm{~g}$ ai ha $^{-1}$ as pre-em & 100.26 & 3.04 & 3.89 & 28.57 \\
\hline $\begin{array}{c}\mathrm{T}_{9} \text { Pretilachlor @ } 1000 \mathrm{~g} \mathrm{ai} \mathrm{ha}^{-1} \text { as pre-em } f b 2,4- \\
\text { D @ } 750 \mathrm{~g} \text { ai ha }{ }^{-1} \text { as post-em }\end{array}$ & 95.89 & 3.04 & 4.33 & 30.66 \\
\hline $\begin{array}{l}\mathrm{T}_{10} \text { Pretilachlor @ } 1000 \mathrm{~g} \text { ai ha }{ }^{-1} \text { as pre-em } f b \\
\text { Bispyribac sodium @ } 20 \mathrm{~g} \text { ai ha }{ }^{-1} \text { as post- em }\end{array}$ & 93.40 & 3.44 & 6.44 & 30.72 \\
\hline $\mathrm{T}_{11}$ Weed free & 109.11 & 3.61 & 4.89 & 32.50 \\
\hline $\mathrm{SEm} \pm$ & 3.72 & 0.16 & 0.31 & 1.08 \\
\hline $\mathrm{CD}(\mathrm{P}=0.05)$ & 10.98 & 0.46 & 0.91 & 3.19 \\
\hline
\end{tabular}

Table 2: Effect of weed management practices on yield attributes of transplanted finger millet

\begin{tabular}{|c|c|c|c|c|c|}
\hline \multirow{2}{*}{ Treatments } & \multicolumn{5}{|c|}{ Yield attributes } \\
\hline & No of ear $\mathrm{m}^{-2}$ & Ear weight (g) & No of finger ear-1 & Finger length $(\mathrm{cm})$ & Test weight $(\mathrm{g})$ \\
\hline $\mathrm{T}_{1}$ Weedy check (Control) & 102.33 & 7.14 & 6.55 & 7.84 & 3.91 \\
\hline $\mathrm{T}_{2}$ Hoeing at 20 and 40 DAT & 119.33 & 8.49 & 7.11 & 8.87 & 4.46 \\
\hline $\mathrm{T}_{3}$ Pendimethalin @ $750 \mathrm{~g}$ ai ha ${ }^{-1}$ as pre-em & 110.00 & 8.02 & 6.78 & 8.26 & 4.34 \\
\hline $\mathrm{T}_{4} 2,4-\mathrm{D} @ 750 \mathrm{~g}$ ai ha ${ }^{-1}$ as post-em & 136.33 & 8.03 & 6.88 & 8.35 & 4.35 \\
\hline $\mathrm{T}_{5}$ Bispyribac sodium @ $20 \mathrm{~g}_{\text {ai ha }}{ }^{-1}$ as post-em & 147.33 & 8.09 & 6.91 & 8.39 & 4.38 \\
\hline $\begin{array}{c}\text { T6 Pendimethalin @ } 750 \mathrm{~g} \text { ai ha }^{-1} \text { as pre-em } f b 2, \\
\text { 4-D @ } 750 \mathrm{~g} \mathrm{ai} \mathrm{ha}^{-1} \text { as post-em }\end{array}$ & 140.33 & 8.18 & 6.95 & 8.46 & 4.51 \\
\hline $\begin{array}{l}\mathrm{T}_{7} \text { Pendimethalin @ } 750 \mathrm{~g} \text { ai ha }{ }^{-1} \text { as pre-em } f b \\
\text { Bispyribac sodium @ } 20 \mathrm{~g} \text { ai ha }{ }^{-1} \text { as post-em }\end{array}$ & 155.67 & 8.22 & 7.00 & 8.54 & 4.41 \\
\hline $\mathrm{T}_{8}$ Pretilachlor @ $1000 \mathrm{~g}_{\text {ai ha }}{ }^{-1}$ as pre-em & 112.67 & 8.15 & 6.77 & 8.45 & 4.37 \\
\hline $\begin{array}{l}\text { T9 Pretilachlor @ } 1000 \mathrm{~g} \mathrm{ai} \mathrm{ha}^{-1} \text { as pre-em } f b 2,4- \\
\text { D @ } 750 \mathrm{~g} \text { ai ha }{ }^{-1} \text { as post-em }\end{array}$ & 115.63 & 8.17 & 6.89 & 8.46 & 4.38 \\
\hline $\begin{array}{l}\mathrm{T}_{10} \text { Pretilachlor @ } 1000 \mathrm{~g} \text { ai ha }{ }^{-1} \text { as pre-em } f b \\
\text { Bispyribac sodium @ } 20 \mathrm{~g}^{\text {ai ha }} \mathrm{ha}^{-1} \text { as post- em }\end{array}$ & 158.67 & 8.26 & 7.07 & 8.58 & 4.43 \\
\hline $\mathrm{T}_{11}$ Weed free & 129.00 & 8.52 & 7.22 & 8.90 & 4.53 \\
\hline SEm \pm & 4.67 & 0.16 & 0.19 & 0.15 & 0.11 \\
\hline $\mathrm{CD}(\mathrm{P}=0.05)$ & 13.77 & 0.48 & 0.56 & 0.45 & 0.31 \\
\hline
\end{tabular}

Table 3: Effect of weed management practices on yield and economics of transplanted finger millet

\begin{tabular}{|c|c|c|c|c|}
\hline \multirow{2}{*}{ Treatments } & \multicolumn{2}{|c|}{ Yield } & \multicolumn{2}{|c|}{ Economics } \\
\hline & Grain yield $\left(\right.$ qha $\left.^{1}{ }^{1}\right)$ & Straw yield $\left(\right.$ qha- $\left.^{-1}\right)$ & Net return $(\mathrm{Rs} / \mathrm{ha})$ & B:C ratio \\
\hline $\mathbf{T}_{1}$ Weedy check (Control) & 16.31 & 33.31 & 32400 & 1.15 \\
\hline $\mathbf{T}_{2}$ Hoeing at 20 and 40 DAT & 23.00 & 43.82 & 43356 & 1.06 \\
\hline $\mathbf{T}_{3}$ Pendimethalin @ $750 \mathrm{~g}$ ai ha ${ }^{-1}$ as pre-em & 18.89 & 37.05 & 40333 & 1.37 \\
\hline $\mathbf{T}_{4} 2,4-\mathrm{D} @ 750 \mathrm{~g} \mathrm{ai} \mathrm{ha}^{-1}$ as post-em & 17.41 & 33.56 & 35485 & 1.24 \\
\hline T5 Bispyribac sodium @ $20 \mathrm{~g}_{\text {ai ha }}{ }^{-1}$ as post-em & 20.61 & 40.10 & 46158 & 1.55 \\
\hline $\begin{array}{c}\text { T6 Pendimethalin @ } 750 \mathrm{~g} \text { ai ha }^{-1} \text { as pre-em } f b 2, \\
\text { 4-D @ } 750 \mathrm{~g} \mathrm{ai} \mathrm{ha}{ }^{-1} \text { as post-em }\end{array}$ & 19.86 & 40.25 & 43921 & 1.48 \\
\hline $\begin{array}{l}\text { T}_{7} \text { Pendimethalin@750 g ai ha }{ }^{-1} \text { as pre-em } f b \\
\text { Bispyribac sodium @ } 20 \mathrm{~g} \text { ai ha }{ }^{-1} \text { as post-em }\end{array}$ & 22.65 & 43.23 & 52242 & 1.69 \\
\hline T8 Pretilachlor @ $1000 \mathrm{~g}$ ai ha ${ }^{-1}$ as pre-em & 18.80 & 36.39 & 39973 & 1.37 \\
\hline $\begin{array}{l}\text { T9 Pretilachlor @ } 1000 \mathrm{~g} \mathrm{ai} \mathrm{ha}^{-1} \text { as pre-em } f b 2, \\
\text { 4- D @ } 750 \mathrm{~g} \text { ai ha }{ }^{-1} \text { as post-em }\end{array}$ & 19.82 & 39.64 & 43730 & 1.48 \\
\hline $\begin{array}{l}\text { T10 Pretilachlor @1000 g ai ha }{ }^{-1} \text { as pre-em } f b \\
\text { Bispyribac sodium @ } 20 \mathrm{~g} \mathrm{ai} \mathrm{ha}^{-1} \text { as post-em }\end{array}$ & 22.59 & 43.46 & 52253 & 1.70 \\
\hline $\mathbf{T}_{11}$ Weed free & 23.12 & 44.01 & 39500 & 0.87 \\
\hline $\mathrm{SEm} \pm$ & 0.75 & 1.23 & 2612 & 0.08 \\
\hline $\mathrm{CD}(\mathrm{P}=0.05)$ & 2.22 & 3.64 & 7706 & 0.25 \\
\hline
\end{tabular}




\section{References}

1. Anonymous. Ministry of Agriculture \&amp; Farmers Welfare, Government of India, -Directorate of Statistical and Evaluation, 2017.

2. Prithvi BK, Rao AS, Srinivasulu K. Weed management in transplanted ragi. 2015; 47(2):214-215.

3. Pradhan A, Rajput AS, Thakur A. Effect of weed management on growth and yield of finger millet. Indian Journal of Weed Science. 2010; 42(1-2):53-56.

4. Satish P, Lakra RK, Nargis K, Alam P, Puran AN. Weed Management on Direct Seeded Finger Millet (Eleusine coracana L.) Under Rainfed Condition of Jharkhand. ISSN: 2319-7706 Special Issue-7, 2018, 844-850.

5. Tuti MD, Singh S, Pandey BM, Bisht JK, Pattanayak A. Weed management in rainfed finger millet. 2016; 48(1):74-75.

6. Shanmugapriya $\mathrm{P}$, Rathika $\mathrm{S}$, Ramesh $\mathrm{T}$, Janaki $\mathrm{P}$. Evaluation of weed management practices on weed control and yield of transplanted finger millet. 2019; 8(5):276-278.

7. Pradhan A, Rajput AS, Thakur A. Effect of weed management practices on finger millet under rainfed conditions. Indian Journal of weed Science. 2012; 44(2):115-117.

8. Kujur S, Singh VK, Gupta DK, Tandon A, Ekka V, Agrawal HP. Influence of Weed Management Practices on Weeds, Yield and Economics of Fingermillet (Eleusine coracana L. International Journal of Bioresource and Stress Management. 2018; 9(2):209-213.

9. Naik DC, Muniyappa TV, Kumar MD. Effect of integrated weed management on nutrient uptake transplanted ragi and associated weeds. 2000; 13(4):819823.

10. Prashanth Kumar MK, Shekara BG, Yamuna BG, Sunil CM. Crop Weed Competition for Nutrients by Weed and Drill Sown Finger millet (Eleusine coracana L. Gaertn.). National Academy of Agriculture Science. 2015; 33(3):2049-2054.

11. Nanjappa HV, Hosmani MM, Prabhakara Setty TK. Nutrient uptake by weeds as influenced by crop weed competition under different cropping systems in finger millet. Mysore Journal of Agricultural Sciences. 1987; 21:140-144. 